\title{
TRIANGULATING SOUND SYMBOLISM: WHERE TO FIND IT AND HOW TO CREATE IT
}

\author{
Niklas Johansson* \\ *Corresponding Author: niklas.johansson@ling.lu.se \\ Centre for Languages and Literature, Lund University, Lund, Sweden
}

Sound symbolism is a universal component of language (Samarin 1978; Blasi et al. 2016), but it can also adapt to language-specific constraints such as phoneme inventories, since different languages often use various, albeit phonetically similar, types of sounds for the same sound symbolic association. It is thus natural to investigate the phenomenon from a bottom-up perspective without any initial assumptions other than that it is a universal, non-arbitrary and flexible association between sound and meaning. However, most previous crosslinguistic studies have been small in scope, and larger-scale studies (Wichmann et al. 2010; Blasi et al. 2016) have not captured many phonetic distinctions important for sound symbolism, e.g. voicing (Ohala 1994; Johansson 2017). Furthermore, experiments have usually focused on matching ready-made sound symbolic words to different stimuli (cf. Ramachandran \& Hubbard 2001), rather than investigating how sound symbolic associations develop among language users. The present study attempts to amend these issues by focusing on how sound symbolism operates through a more thorough examination of the phonetic and semantic features involved, both cross-linguistically and experimentally.

First, 344 concepts with claimed universal tendencies (e.g. Swadesh 1971; Goddard and Wierzbicka 2002) were investigated in 245 language families and the phonemes of the linguistic forms were systematically grouped according to phonetically salient parameters to pinpoint the features responsible for each sound symbolic association. 178 statistically significant sound-meaning associations were found based on the standard scores calculated for the occurrence of each sound group in each concept, and their occurrence in all of six geographical macro-areas. In addition, these associations could in turn be correlated with at least 45 out of the 100 items of the regular Swadesh-list (Swadesh 1971), raising several questions about the validity of the list as a tool for establishing genetic relationships. Secondly, 42 macro-concepts were 
identified based on cooccurring shared semantic and phonetic features between the significant concepts. Most of these had basic descriptive functions (HARD, SMALL, DARK, UNEVEN, etc.), but also included deictic distinctions and kinship attributes. Furthermore, all identified macro-concepts were found to be grounded in one or several of four types of sound symbolism (cf. Dingemanse 2011; Carling \& Johansson 2015): (a) in unimodal imitation, or onomatopoeia, based on auditory similarity; (b) in a more indirect and cross-modal type of imitation which is grounded in similarities between the referent and vocal gestures, in which the accompanying sounds are only secondarily associated with the meaning (e.g. ROUND and labial sounds which have visually round shapes); (c) in the frequency code (Ohala 1994), in which resemblance is based on relation with both indexical and iconic grounds; or in (d) an even weaker type of sound symbolism, based on pure circumstantial, indexical associations, e.g. the association between MOTHER, MILK, BREAST etc. and nasals, since those are the only sounds that infants are able to produce whilst breastfeeding.

Thirdly, four of the confirmed sound symbolic concepts were further investigated through iterated learning experiments (Kirby et al. 2015). Naïve participants were divided into five condition groups which contained ten chains of 15 participants each. They either received no information about the meaning of the word they were about to hear, or that it meant BIG, SMALL, ROUND or POINTY, which created a meaning-bias. The first participant in each chain was then audially presented with a phonetically diverse word and asked to repeat it. Thereafter, the recording of the repeated word was played for the next participant in the same chain. Significant increases of high frequency sounds and sounds produced using the hard palate in the SMALL- and POINTYconditions, and labial and low frequency in the ROUND-condition, were found after 15 generations. These results further revealed that the continuous SIZEdomain was associated with pitch, while the dichotomous SHAPE-domain was associated with the use of separate tactile and visual vocal gestures.

These findings show considerable cross-linguistic sound symbolic effects on basic vocabulary regardless of language family, and that sound symbolism evidently still is an active part of language. They also illustrate how sound symbolism is based in the human perception of the body and its interaction with the surrounding world which is associated through several types of iconicity with different degrees of directness. Thus, it is likely that sound symbolism has originated as a bootstrapping mechanism (Imai \& Kita 2014) and could have had an even more crucial communicative role in earlier stages of human language. 


\section{References}

Blasi, D. E., Wichmann, S., Hammarström, H., Stadler, P. F., \& Christiansen, M. H. (2016). Sound-meaning association biases evidenced across thousands of languages. Proceedings of the National Academy of Sciences.

Dingemanse, M. (2011). Ezra Pound among the Mawu: Ideophones and Iconicity in Siwu. In P. Michelucci, O. Fischer and C. Ljungberg (Eds), Iconicity in Language and Literature, 15, 39-54. Amsterdam: John Benjamins.

Goddard, C. \& Wierzbicka, A. (eds.) (2002). Meaning and Universal Grammar: Theory and Empirical Findings (2 volumes). Amsterdam/Philadelphia: John Benjamins.

Imai, M., \& Kita, S. (2014). The sound symbolism bootstrapping hypothesis for language acquisition and language evolution. Phil. Trans. $R$. Soc. $B, 369(1651)$.

Johansson, N. (2017). Tracking linguistic primitives: The phonosemantic realization of fundamental oppositional pairs. In: Matthias Bauer, Angelika Zirker, Olga Fischer \& Christina Ljungberg (eds.) Dimensions of Iconicity. Iconicity in Language and Literature 15. Amsterdam: John Benjamins.

Johansson, N. \& Carling, G. (2015). The De-Iconization and Rebuilding of Iconicity in Spatial Deixis: A Indo-European Case Study. Acta Linguistica Hafniensia: International Journal of Linguistics, 47:1, 4-32.

Kirby, S., Tamariz, M., Cornish, H., \& Smith, K. (2015). Compression and communication in the cultural evolution of linguistic structure. Cognition, 141, 87-102.

Ohala, J. J. (1994). The frequency code underlies the sound-symbolic use of voice pitch. In L. Hinton, J. Nichols and J.J. Ohala (Eds), Sound symbolism, 325-347. Cambridge: Cambridge University Press.

Ramachandran, V. S., \& Hubbard, E. M. (2001). Synaesthesia - a window into perception, thought and language. Journal of consciousness studies, 8(12), 334.

Samarin, W. (1978) Linguistic adaptation to speech function. In W. Mc Cormack and S. A. Wurm (Eds), Approaches to language: Anthropological issues. Mouton: The Hague.

Swadesh, M. (1971). The origin and diversification of language. Edited post mortem by Joel Sherzer. London: Transaction Publishers.

Wichmann, S., Holman, E. W. \& Brown, C. H. (2010). Sound Symbolism in Basic Vocabulary. Entropy 12(4), 844-858. 